\title{
The role of user emotional attachment in driving the engagement of children with Au- tism Spectrum Disorders (ASD) in using a smartphone app designed to develop social and life skill functioning
}

\author{
Dr Joseph Mintz \\ Institute of Education, University of London, 120 Bedford Way, London WC1H 0LB \\ Email: j.mintz@ioe.ac.uk
}

\begin{abstract}
There has been, in the last ten years, a fast developing interest in the potential use of mobile technology in the classroom and in particular, in the use of such technology to support children with ASD (Autistic Spectrum Disorders). The HANDS project developed a software application for mobile Smartphones based on the principles of persuasive technology design, which supports children with ASD with social and life skills functioning - areas of ability which tend to be impaired in this population. Issues with the application of a behaviorist approach to the complex social field of special educational needs are considered. An argument is made for the need for 'thick' persuasive applications which take account of such complexity including the role of mediating factors. Particular focus is given to qualitative results indicating that user emotional attachment is one such key factor.
\end{abstract}

Keywords. User Emotional Attachment; Autism; Special Educational Needs, Instructional Design, Persuasive Technology

\section{$1 \quad$ Introduction}

Impairments in social and communicative reciprocity and in adaptive, flexible regulation of self and behaviors in individuals with ASD lead to significant difficulties in social participation both in simple situations, such as shopping and catching a bus to school as well as more intensely in complex social transactions, such as building relationships, or fitting in with classroom rules in educational contexts [1]. Perhaps unsurprisingly, there has been some hope invested in the potential of assistive technology, and particularly since the launch of the iPhone in 2007, in the use of mobile technology to support individuals with ASD in overcoming these barriers to social interaction. In an exploratory study, Mechling et al. [2] evaluated the use of a mobile device with multiple prompt levels, indicating its potential to increase efficacy in completion of novel tasks and transitioning within and between tasks. Gentry et. al. [3] 
used standardized measurement tools to evaluate the efficacy of mobile devices as cognitive aids in a sample of high school students with ASD, indicating positive initial outcomes. Reports have also being published on the use of mobile technology specifically to support social skills development. For example, Tentori and Hayes [4] report on the initial implementation of a smartphone app designed to give children social cues in specific social situations. Such research findings form the basis of support for the development and systematic evaluation of a fully integrated and flexible cognitive support tool on a mobile smartphone which can offer a wide range of interventions personalized to the individual needs of a student with ASD. More recently, a number of small scale studies, such as Neely et al. [5] and Mechling, Foster and Ayres [6], have been published on the use of tablet technology for similar purposes.

One such system was developed in the HANDS (Helping Autism Diagnosed Navigate and Develop Socially) research project, which created a cognitive support smartphone app which allows teachers to flexibly develop interventions on such devices to support children with ASD in developing social and life skills. The software design for this system was based on the principles of Persuasive Technology [7]. This paper reports on aspects of the use and evaluation of the HANDS system.

\section{Persuasive Technology}

The initial development of persuasive design principles by Fogg [7] was rooted in social psychology, but also influenced by behaviorism, cognitive psychology and communication theory. Fogg also drew on the Computers as Social Actors Approach developed by Nass et. al. [8] in which a social rule or dynamic for human-human interaction is adapted so that instead of two humans interacting, we have a computer and a human interacting in a social context. There has been considerable use and development of persuasive systems in healthcare and commerce/marketing (see [9] for recent examples).

Fogg [7] highlights several specific design principles for bringing about behavior change that are particularly well suited to incorporation in to technology systems:

1. Reinforcement - reinforce target behaviors positively when they happen or negatively when they don't

2. Reduction - make a complicated task simpler by removing stages in the process

3. Self monitoring - allow the user to monitor their behavior

4. Suggestion - perform an intervention at the most opportune moment when the user has both motivation and the ability to act.

5. Surveillance - the person's behavior is monitored and observed by other people

6. Tailoring - use of personally relevant information as part of the intervention.

7. Tunneling - sequencing tasks, to reduce cognitive load on the user, and thus support completion of the overall goal. 


\subsection{Credibility and Kairos}

Fogg also makes extensive use of the two other concepts - source credibility and Kairos. The former is typically categorized in the literature as having two dimensions - trustworthiness and expertise [10]. Trustworthiness indicates the perceived goodness or morality of the source and expertise indicates the perceived knowledge and skill of the source. Tseng and Fogg [11] consider specific features which can influence perceived computer credibility, including interface design features such as color tones and balanced layout. Fogg regards credibility as a central issue for the design of persuasive systems because the inherent inflexibility of computer systems means that design errors in this area can very easily lead to irretrievable losses of perceived source credibility.

Fogg also highlights the concept of Kairos, namely that messages are more likely to be persuasive if they are delivered at the right time and place. Fogg [7, 12] considers Kairos as being particularly relevant for mobile persuasive systems. He argues that as mobile phones, integrated with social networking technology, become more and more a central part of the lives of students, mobile offers opportunities for interventions to be delivered at a time and place when they are receptive to receiving them.

\subsection{The HANDS System}

Modeling the HANDS software on persuasive technology design resulted in development of the following key functionalities: 1) an interactive calendar function with prompts, 2) a "Personal Trainer" training and support function that allowed teachers to flexibly develop specific interventions including step by step guidance sequences incorporating video and audio content. These interventions were designed on a server based application and then uploaded to the client application on the child's smartphone. The Personal Trainer is the key arena for the expression of persuasive design principles in the software, further exploiting persuasive principles including tailoring to the specific needs of the individual, personalization (such as choosing the audio and visual skin of the app), reduction (step by step instructions that reduce cognitive load), credibility, and rewards. The first prototype of this software was implemented during the 2010 school year. Following a training programme, teachers worked with individual children to develop tailored interventions using the smartphone app, with ongoing support from the academic and technical team. Evaluation of this prototype was fed in to the development of the second prototype which was similarly implemented in the 2011 school year. Implementation took place at four special schools across Europe and involved 20 teachers and 40 children.

\section{Special Educational Needs and Persuasive Technology}

From one perspective, special educational needs, such as Autism, Attention Deficit Hyperactivity Disorder, Oppositional Defiant Disorder, Down's Syndrome etc. are synonymous with medical categories. Autism is a psychiatric (i.e. medical) definition, conceived primarily as a developmental disorder of the human nervous system. Psy- 
chiatric definitions of autism are clearly located firmly within a medical model (see Barton 1988's seminal discussion [13]), and as such, when introduced in to the social field of education have been subjected to sociological critiques which note that 'normal' modes of social and emotional communication can be thought of as socially constructed, and that greater recognition might be given, in educational setting in particular, to seeing this is difference rather than deficit, and that as such the diagnostic labels themselves could even be considered as negatively pathologizing groups of children $[14,15]$. These two accounts (psychiatric and sociological) are in fact often both extant, frequently with considerable tension, in the minds of teachers and in the national and local discourses and debates on policy on special educational needs.

This discussion is of particular relevance to the use of persuasive technology for special educational needs because of persuasive technology's provenance. As discussed, Fogg brought together these strands from the existing disciplines of rhetoric, social psychology and persuasion research in his 'new' discipline of persuasive technology. However, the behaviorist slant of Fogg's work is clear. It is reasonable to postulate that there is a tension which arises when an approach derived from behaviorism (although certainly not in Fogg's case a naïve variety) is applied to special educational needs, a field where the debates between medical versus social conceptualizations of the object of activity put in to question the linear relationship between action and object that a behaviorist approach implies.

This analysis points towards the complexity of the social field in to which persuasive applications are applied. In 'traditional' persuasive technology, applications are designed to apply to a tightly defined area of behavior, i.e. to persuade a person to undertake one clearly specified behavior in one clearly specified context. For example, Kim, Hong, and Magerko [16] discuss a comparative study of the effectiveness of two PC-based widget applications, each designed to give a visual metaphor for the energy consumption when the PC is on but not in use, thus persuading users to switch their PC off when not being used. Thus we have a clearly specified behavior - switch your PC off when you are not using it, in a clearly specified context; that is, the use of your PC. In addition, 'traditional' applications are relatively context free; that is, there is very limited information available about individual target users, and thus applications focus on interventions and persuasive messages that can be easily replicated for use across large populations with a low level of individualization. Although tailoring has been identified as a persuasive strategy, the implementation of this strategy in typical persuasive applications generally uses a very limited data set about individual users. In educational contexts the area of behavior targeted as well as the context are typically diffuse. Persuasive technology design to date has resided outside of a defining social context and empirical research has paid attention to persuasive effects rather than studying and using the social context. Yet when you are discussing persuasive effects in relation to special educational needs, where even the terms are contested and complex, persuasion needs to be considered differently. However, I argue that it is perfectly possible to develop what can be termed "thick" persuasive applications which explicitly take account of the social context and can be applied to a more complex range of behaviors such as those related to special educational needs in the classroom. Where then does the potential benefit of persuasive design for special educa- 
tional needs lie? In many diagnostic conditions, including autism, ADHD, Oppositional Defiant Disorder, Developmental Coordination Disorder, we see impairments in social communication, emotional regulation and social interaction, linked to difficulties with adaptive, flexible regulation of self and behaviors. Consequently, a major objective of the education of young people with these conditions is the modification of behaviors associated with impairments in social and life skill functioning [17]. Thus persuasive technology has the potential to help children with special educational needs who want to change their behavior (and one of Fogg's key principles [7] is that persuasion via technology is only effective when the 'target recipient' wants to change their behavior on some level). However, 'thick' applications need to take account of the complex social field, one aspect of which is that whether a persuasive message delivered by technology is effective depends on how it is mediated in that social field. One of those factors is user emotional attachment.

\section{User Emotional Attachment}

Writing from a general HCI perspective, Meschtscherjakov [18] and Wehmeyer [19] have noted that mobile device users develop a relationship with their mobile devices and that their level of engagement with the device generally and with particular functions is dependent on the quality of that relationship. They use the terms "user attachment" or "user emotional attachment" to signify that mobile devices can act as an expression of an individual's personality or a symbol for group membership, and as such act as an extension of or form part of an individual's sense of identity. Wehmeyer [19] and later Geven et. al. [20] note that personalization (i.e. the tailoring of mobile phone functions such as wallpaper, screensavers etc.) can be one aspect of the process whereby users become "attached" to their mobile devices on an emotional level. Fogg and Eckles [12] use the analogous term "mobile marriage", which is the development of an intensive positive relationship between the user and the device, based on repeated interactions over a period of time. Fogg and Eckles also employ the term "mobile loyalty", which is the perception by users that a device exists to serve their needs and wishes first. The existence of such a perception is postulated as increasing the level of credibility of the persuasive messages provided by on the device, credibility being a key principle of persuasive design. Clearly both concepts are interdependent, as a user who has developed a positive emotional attachment to their device via repeated interaction (user device attachment) is potentially more likely to view persuasive messages on the device as being more credible .

\section{$5 \quad$ Research Methodology}

The objectives of the interpretivist qualitative analysis reported on here were to gain an understanding of the factors mediating engagement with the app. A linked quantitative analysis measuring the extent to which the software had an impact on developing social and life skills is reported elsewhere [21]. Classroom observations and linked semi-structured interviews were carried out with 15 teachers working with 
children using the app using the across the four test school sites, and a linked questionnaire was completed by an additional 15 teachers. Interviews were also undertaken with 18 children across the four schools, and with 16 parents.

\section{Data Analysis}

A thematic analysis of the full data set was undertaken. This followed Fereday \& Muir-Cochrane [22] in using a combined deductive / inductive procedure for the coding of the data facilitated by the Nvivo software package. Identification of themes and patterns in the data was based on a descriptive cross case comparison [23, 24]. Each instance of a teacher working with a child (teacher-child dyad) was considered as a case. Firstly a case study summary was constructed for each case, with emerging themes and patterns being highlighted. Then each case was iteratively compared to the others, and the identified themes and patterns modified during this process to ensure a best fit to the overall data set.

\section{$7 \quad$ Results and Discussion}

The key factors mediating engagement (the 'thick' aspects of persuasive technology use with this group of children with special needs) are indicated in Table 1.

Table 1. Factors Mediating Engagement

\begin{tabular}{|c|c|c|}
\hline Factor & Number of Cases +ve & Number of Cases -ve \\
\hline Technical factors & 14 & 15 \\
\hline $\begin{array}{l}\text { Graphical User Interface } \\
\text { Design }\end{array}$ & 6 & 11 \\
\hline Source Credibility & 13 & 0 \\
\hline User Device Attachment & 18 & \\
\hline Kairos & 23 & 0 \\
\hline $\begin{array}{l}\text { Home-School Collabora- } \\
\text { tion }\end{array}$ & 20 & 0 \\
\hline
\end{tabular}

Cases are +ve where the influence on engagement was positive and-ve where the influence on engagement was negative

As indicated in Table 1, user emotional attachment was identified as a mediating factor in 18 cases (see Mintz et. al. [21]) for a discussion of other factors identified). Teacher, child and parent interviews and teacher questionnaires demonstrated high levels of individual usage of the mobile functions and the width and breadth of functions that the children used, and that in the perception of teachers and parents, broader engagement with these wider phone functions influenced the children's level of engagement with the intervention app and responsiveness to persuasive messages designed to develop social and life skill functioning. 
The qualitative analysis gives some support to the proposition that user device attachment is a relevant factor influencing how children using persuasive mobile systems engage with their device and with specific persuasive functions. Certainly it seems clear that overall attachment is influenced, as suggested in the persuasive design literature, by repeated positive interactions with a range of cognitive and social functions on the device.

This paper also lends support to the contention that thick persuasive applications, which take account the complex social field which children with special educational needs, their parents and their teachers inhabit, could have a positive effect on behavior change. However, for this to happen, consideration of relevant mediating factors, in other words, how these interventions are assimilated in to the world of the child and made meaningful for the user is crucial. User emotional attachment could be conceived of as a conceptualization of the point that children with autism (and likely with ADHD, ODD etc.) do enter in to a type of emotional connection with their device, and it is this emotional connection to the whole device, rather than to just a persuasive app, which is meaningful for them. Persuasive technology may have behaviorist roots, but when it takes proper account of what technology means for individual children, and the complexities that are involved for teachers in working with such children, it can have a role to play with children with special educational needs. Such a conclusion also implies that qualitative research methods as applied here are crucial in letting us uncover those meanings.

\section{References}

1. Howlin, P.: Outcome in Autism Spectrum Disorders. Handbook of Autism and Pervasive Developmental Disorders. pp. 201-220. Wiley, Hoboken, NJ US (2005).

2. Mechling, L.C., Gast, D.L., Seid, N.H.:Using a personal digital assistant to increase independent task completion by students with autism spectrum disorder. J. Autism Dev. Disord. 39, 1420-34 (2009).

3. Gentry, T., Wallace, J., Kvarfordt, C., Lynch, K.B.: Personal digital assistants as cognitive aids for high school students with autism : Results of a community-based trial. J. Vocat. Rehabil. 32, 101-107 (2010).

4. Tentori, M., Hayes, G.: Designing for interaction immediacy to enhance social skills of children with autism. In: Bardram, J., Langhenreich, M., Truong, K., and Nixon, P. (eds.) Proceedings of the 12th ACM international conference on Ubiquitous computing. pp. 51-60. ACM Press (2010).

5. Neely, L., Rispoli, M., Camargo, S., Davis, H., Boles, M.: The effect of instructional use of an iPad on challenging behavior and academic engagement for two students with autism. Research in Autism Spectrum Disorders. 7, 509-516 (2013).

6. Mechling, L.C., Foster, A.M., Ayres, K.M.: Navigation Between Menu Screens and Multiple Touch Points on a Touch Screen Tablet to Access and Complete Multi-Step Tasks Using Video Prompting. Inclusion. 1, 121-132 (2013). 
7. Fogg, B.J.:Persuasive Techonology. Using Computers to Change what We Think and Do. Morgan Kaufman Publishers, San Francisco, CA (2003).

8. Nass, C., Steuer, J., Tauber, E.R.: Computers are social actors. Conference companion on Human factors in computing systems - Computer Human Interaction '94. pp. 72-28. ACM Press, New York (1994).

9. Berkovsky, S., Freyne, J. :Persuasive Technology: 8th International Conference, Persuasive 2013. Proceedings. Lecture Notes in Computer Science, Volume 7822 2013,. Springer, Sydney, NSW, Australia (2013).

10. McGinnes, E., Ward, C.: Better liked than right: trustworthiness and expertise in credibility. Personal. Soc. Psychol. Bull. 6, 467-472 (1980).

11. Tseng, S., Fogg, B.J.: Credibility and computing technology. Communications of the ACM. 42, 39-44 (1999).

12. Fogg, B.J., Eckles, D.: Mobile Persuasion: 20 Perspectives on the Future of Behavior Change. Stanford Captology Media, Stanford (2007).

13. Barton, L.:The Politics of special educational needs. Routledge, London (1988).

14. Molloy, H., Vasil, L.:The Social Construction of Asperger Syndrome: The pathologising of difference? Disabilty and Society 17, 659-669 (2002).

15. Bogdashina, O.:Theory of mind and the triad of perspectives on autism and Asperger syndrome: a view from the bridge. Jessica Kingsley Publishers, London (2006).

16. Kim, T., Hong, H., Magerko, B.: Designing for persuasion: toward ambient eco-visualization for awareness. Persuasive technology 2010. pp. 106-116. Springer (2010).

17. Jordan, R.: Managing autism and Asperger's syndrome in current educational provision. Pediatric Rehabilitation 8, 104-112 (2005).

18. Meschtscherjakov, A.: Mobile attachment - Emotional attachment towards mobile devices and services. The Proceedings of the 11th International Conference on Human-Computer Interaction with Mobile Devices and Services, Mobile HCI'09. , Bonn, Germany (2009).

19. Wehmeyer, K.: Assessing users' attachment to their mobile devices. The Proceedings of the 6th International Conference on Mobile Business, ICMB'07. IEEE Computer Society, Toronto, Canada (2007).

20. Geven, A., Schrammel, J., Tscheligi, M., Mayer, M.: Cell phone design for teenage use. In: Cunliffe, D. (ed.) HCI '08 Proceedings of the Third IASTED International Conference on Human Computer Interaction. pp. 236-241. ACTA Press, Anaheim, USA (2008).

21. Mintz, J., Gyori, M., Aagaard, M.: Touching the Future Technology for Autism?: Lessons from the HANDS Project. IOS Press, Amsterdam (2013).

22. Fereday, J., Muir-cochrane, E.: Demonstrating Rigor Using Thematic Analysis : A Hybrid Approach of Inductive and Deductive Coding and Theme Development. International Journal of Qualitative Methods. 5, 1-11 (2006).

23. Yin, K.: The Case Study Crisis: Some Answers. Adminstrative Science Quarterly 26, 58-65 (1981).

24. Sada, A.N., Maldonado, A.: Research Methods in Education. Sixth Edition by Louis Cohen, Lawrence Manion and Keith Morrison. Taylor \& Francis, (2007) 